\title{
A yeast model for the study of human DFNA5, a gene mutated in nonsyndromic hearing impairment
}

\author{
Juraj Gregan $^{\mathrm{a}, *, 1}$, Lut Van Laer ${ }^{\mathrm{b}}$, Louis D. Lieto ${ }^{\mathrm{c}, 2}$, Guy Van Camp ${ }^{\mathrm{b}}$, Stephen E. Kearsey ${ }^{\mathrm{a}}$ \\ ${ }^{a}$ Department of Zoology, University of Oxford, South Parks Road, Oxford OXI 3PS, UK \\ ${ }^{\mathrm{b}}$ Department of Medical Genetics, University of Antwerp, Antwerp, Belgium \\ ${ }^{\mathrm{c}}$ Department of Veterinary Science, University of Kentucky, Lexington, KY, USA
}

Received 30 January 2003; received in revised form 8 May 2003; accepted 14 May 2003

\begin{abstract}
A mutation in human DFNA5 is associated with autosomal dominant nonsyndromic hearing impairment. The function of DFNA5 protein remains unknown and no experimental model has been described so far. Here we describe fission yeast Schizosaccharomyces pombe as a model organism for studying the function of heterologously expressed DFNA5. We have expressed wild-type as well as mutant DFNA5 alleles under control of regulatable $n m t 1$ promoter. Yeast cells tolerated expression of wild-type DFNA5, while expression of the mutant DFNA5 allele, which is responsible for nonsyndromic autosomal dominant hearing impairment, led to cell cycle arrest. We identified new rat and horse DFNA5 homologues and we describe a domain of homology shared between DFNA5 and the Mcm10 family of DNA replication proteins. Genetic interactions between heterologously expressed DFNA5 and a fission yeast $c d c 23$ ( mcm 10) mutant support a possible link between DFNA5 and Mcm10 proteins.
\end{abstract}

(C) 2003 Elsevier Science B.V. All rights reserved.

Keywords: Cell cycle; MCM10; Schizosaccharomyces pombe; Hearing impairment; DFNA5; Yeast

\section{Introduction}

Hearing impairment is the most frequent sensory handicap. Most of the childhood to early adulthood onset forms of nonsyndromic hereditary hearing impairment exhibit an autosomal dominant inheritance pattern and are indicated by DFNA. To date, more than 90 chromosomal loci for nonsyndromic hearing impairment have been mapped to the human genome (Hereditary Hearing Loss Homepage, Van Camp and Smith, World Wide Web URL: http://www.uia. ac.be/dnalab/hhh).

Human DFNA5 (ICERE-1) is one of the genes associated with nonsyndromic autosomal dominant hearing impairment. An insertion/deletion mutation in intron 7 of this gene causes skipping of exon 8 , resulting in premature termination

\footnotetext{
* Corresponding author. Tel.: +44-1865-271-230; fax: +44-1865-271-

E-mail address: juraj.gregan@zoology.oxford.ac.uk (J. Gregan).

${ }^{1}$ Present address: IMP, Dr. Bohr-Gasse 7, A-1030, Vienna, Austria.

${ }^{2}$ Present address: NIH, NIAID, LAD, Rockville, MD, USA.
}

of the open reading frame [1]. In addition to its relevance to hearing impairment, the expression pattern of human DFNA5 indicated that this gene might participate in the tumor biology of breast and skin cancers $[2,3]$. The physiological function of the DFNA5 protein remains unknown and computational analysis did not give any information on the protein function [1]. In addition, no significant homology with any other protein could be detected. Here we report that DFNA5 shares sequence similarity with the Mcm10 family of DNA replication proteins.

The Mcm10 (dna43) gene was first identified in yeast Saccharomyces cerevisiae in two genetic screens for genes required for chromosomal DNA replication and mini-chromosome maintenance [4,5]. Mcm10 is required for efficient initiation of DNA replication. Mcm10 may also be required for the elongation step of DNA replication [6]. Recent observations showed that $\mathrm{Mcm} 10$ performs its function after prereplicative complex assembly and is required for loading of Cdc45 onto chromatin [7] (J. Gregan and S.E. Kearsey, unpublished data). All known members of the Mcm10 family share a conserved zinc-finger-like motif, referred to as the $\mathrm{CCCH}$ domain [8], which is essential for the $\mathrm{Mcm} 10$ 


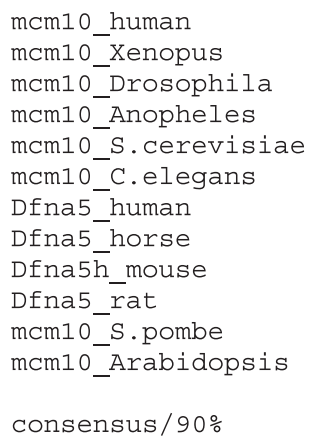

consensus / $90 \%$

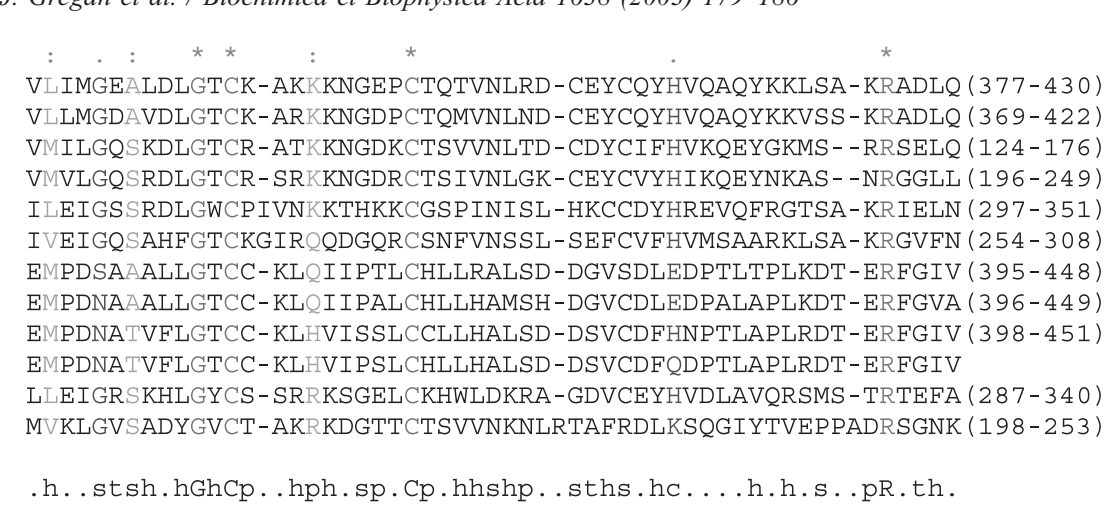

Fig. 1. DFNA5 and Mcm10 proteins share a domain of homology. Multiple alignment of the Mcm10-CCCH domains of human (Swiss-Prot: Q9H3P9), Xenopus laevis (Swiss-Prot: Q9DEX0), Drosophila melanogaster (GenBank: NP_610097), Caenorhabditis elegans (Swiss-Prot: Q9U2C2), Anopheles gambiae (GenBank: EAA10332), Arabidopsis thaliana (GenBank: NP_179694), Schizosaccharomyces pombe (Swiss-Prot: O42709) and Saccharomyces cerevisiae (Swiss-Prot: P32354) and of DFNA5 proteins from human (Swiss-Prot: O60443), mouse (Swiss-Prot: Q9Z2D3), horse (GenBank: AY194290) and rat (GenBank: AY194291) aligned using CLUSTAL W [22]. Identical residues are indicated by an asterisk, strong and weak conservations based on CLUSTAL $\mathrm{W}$ are indicated by a double dot and single dot respectively. The first and the last aligned residues in each of the sequences are designated on the right. The $90 \%$ consensus shown below the alignment was calculated using Consensus (http://www.bork.embl-heidelberg.de:8080/Alignment/consensus.html) and the following amino acid groupings were used: hydrophobic (h; A,C,F,G,H,I,K,L,M,R,T,V,W,Y), small (s; A,C,D,G,N,P,S,T,V), turnlike (t; A,C,D,E,G,H,K,N,Q,R,S,T), polar (p; C,D,E,H,K,N,Q,R,S,T), charged (c; D,E,H,K,R).

function $[9,10]$. The hallmarks of the $\mathrm{CCCH}$ domain are conserved cysteine residues resembling a zinc-finger motif as well as conserved adjacent glycine and arginine residues (Fig. 1).

\section{Materials and methods}

\subsection{Fission yeast methods}

Media, growth conditions and genetic methods were as described by Moreno et al. [11]. Thiamine was used at $5 \mu \mathrm{g} /$ $\mathrm{ml}$ to repress the $n m t 1$ promoter.

Flow cytometry analysis and microscopy were as described in Ref. [12].

\subsection{Plasmid constructs}

\subsubsection{Horse and rat homologues of DFNA5}

A horse skin library in $\lambda$ ZAP Express [13] and a rat brain library in $\lambda$ TriplEx (Clontech) were plated using standard techniques. A 1500-bp mouse DFNA5 hybridization probe was PCR-generated using primers 5'-GTTGATGCTGGAGGAGACC- $3^{\prime}$ and 5'-GTCTTGACCTGTAGCATGTCC- $3^{\prime}$ and the previously cloned mouse Dfna $5 h$ as template [1]. The probe was purified with the QIAquick Gel Extraction Kit (Qiagen). Membranes resulting from plaque lifting were hybridized with the ${ }^{32} \mathrm{P}$-labelled probe using standard techniques. Positive clones were rescreened to purity and phagemid was excised according to the manufacturer's guidelines. Inserts were sequenced using the Big-Dye DyeTerminator Cycle Sequencing Kit (ABI). Fragments were separated on an ABI 3100 automated DNA sequencer (ABI).

After isolation of the partial rat cDNA, a rat-specific probe was generated using PCR primers 5'-GGAGCTTCC-
GTTCCTTTGC-3' and 5'-CAGCAGTGTTGCTGGTGTGC- $3^{\prime}$. In addition, 5'-RACE experiments were performed on the rat library using a rat DFNA5-specific primer $5^{\prime}$ CCCAGCATTGTACAGCATGTCCAAAGG- $3^{\prime}$ and a TriplEx vector primer 5'-CTCGGGAAGCGCGCCATTGTGTTGG-3'.

\subsubsection{Cloning of human full-length and mutant DFNA5}

To obtain a human full-length DFNA5 clone, a PCR reaction was performed on Human Leukocyte MarathonReady cDNA (Clontech) using the Advantage cDNA PCR kit (Clontech) and the following primers: $5^{\prime}-\mathrm{CCG}-$ ACATCTCCCGGATAATCTGG-3' ${ }^{\prime}$ and $5^{\prime}$-GGTCAACTTTTAACGTGCATATGACC- $3^{\prime}$. The PCR reaction consisted of 35 cycles of 30 -s denaturation at $94{ }^{\circ} \mathrm{C}$ and 4 -min extension at $68{ }^{\circ} \mathrm{C}$. The PCR product was cloned into the pT-Adv vector using the AdvanTAge PCR cloning kit (Clontech).

To obtain a human mutant DFNA5 clone, total RNA was isolated from EBV-transformed lymphoblastoid cell lines derived from DFNA5 patients [1] using Trizol (Life Technologies). cDNA was prepared using random hexamers from the SuperScript Preamplification System (Life Technologies). A similar PCR reaction as described above was performed. The resulting PCR fragments were cloned into the pCR4-TOPO vector using the TOPO TA Cloning kit (Invitrogen), and colonies that contained mutant DFNA5 were selected.

Both the human full-length and the mutant DFNA5 clones were completely bidirectionally sequenced in order to exclude the introduction of cloning errors.

Mouse $D f n a 5 h$ was PCR amplified from the pBluescriptDfna5h using oligonucleotide primers 5'XhoImouseDFNA5 (ATATCTCGAGATGTTTGCCAAAGCAACTC) and 3'SmaImouseDFNA5 (ATATCCCGGGCTAGTCTTGACC- 
TGTAG) and cloned into XhoI and $S m a I$ sites of the plasmid pREP3x, resulting in pREP3x-mDFNA5.

Human DFNA5 was excised from the plasmid pT-AdvhDFNA5 by BamHI and SmaI and cloned into BamHI and SmaI sites of the plasmid pREP3x, resulting in pREP3xhDFNA5. Mutant DFNA5 was excised from the plasmid pCR4-hDFNA5 ${ }^{\text {mut }}$ by EcoRI and cloned into the EcoRI site of the plasmid pT-Adv-hDFNA5, replacing the wild-type DFNA5 allele and resulting in pT-Adv-hDFNA5 ${ }^{\text {mut }}$ plasmid. Mutant DFNA5 was excised from the plasmid pT-AdvhDFNA $5^{\text {mut }}$ by BamHI and HincII and cloned into the BamHI and SmaI sites of the plasmid pREP3x, resulting in $\mathrm{pREP} 3 \mathrm{x}-\mathrm{hDFNA} 5^{\mathrm{mut}}$ plasmid. All constructs were verified by sequencing.

\subsection{Yeast strains}

Degron-cdc23-M36 strain P1146 (cdc23-M36-td) was constructed by $B g l$ II linearization of the plasmid pSMRG $2+$ degron $+\operatorname{cdc} 23$ (J. Gregan and S.E. Kearsey, unpublished data) and integration at the $c d c 23$ locus of the Schizosaccharomyces pombe strain P155 (cdc23-M36 $h^{-}$), which contained $c d c 23-M 36$ temperature-sensitive allele. A similar strategy was used to construct degron$c d c 23-I E 2$ strain P1145 (cdc23-IE2-td), where pSMRG2 + degron + cdc23 plasmid was integrated at the $c d c 23$ locus of the $S$. pombe strain P893 (cdc23-IE2 leu1-32 $h^{-}$), which contained the $c d c 23-I E 2$ temperature-sensitive allele.

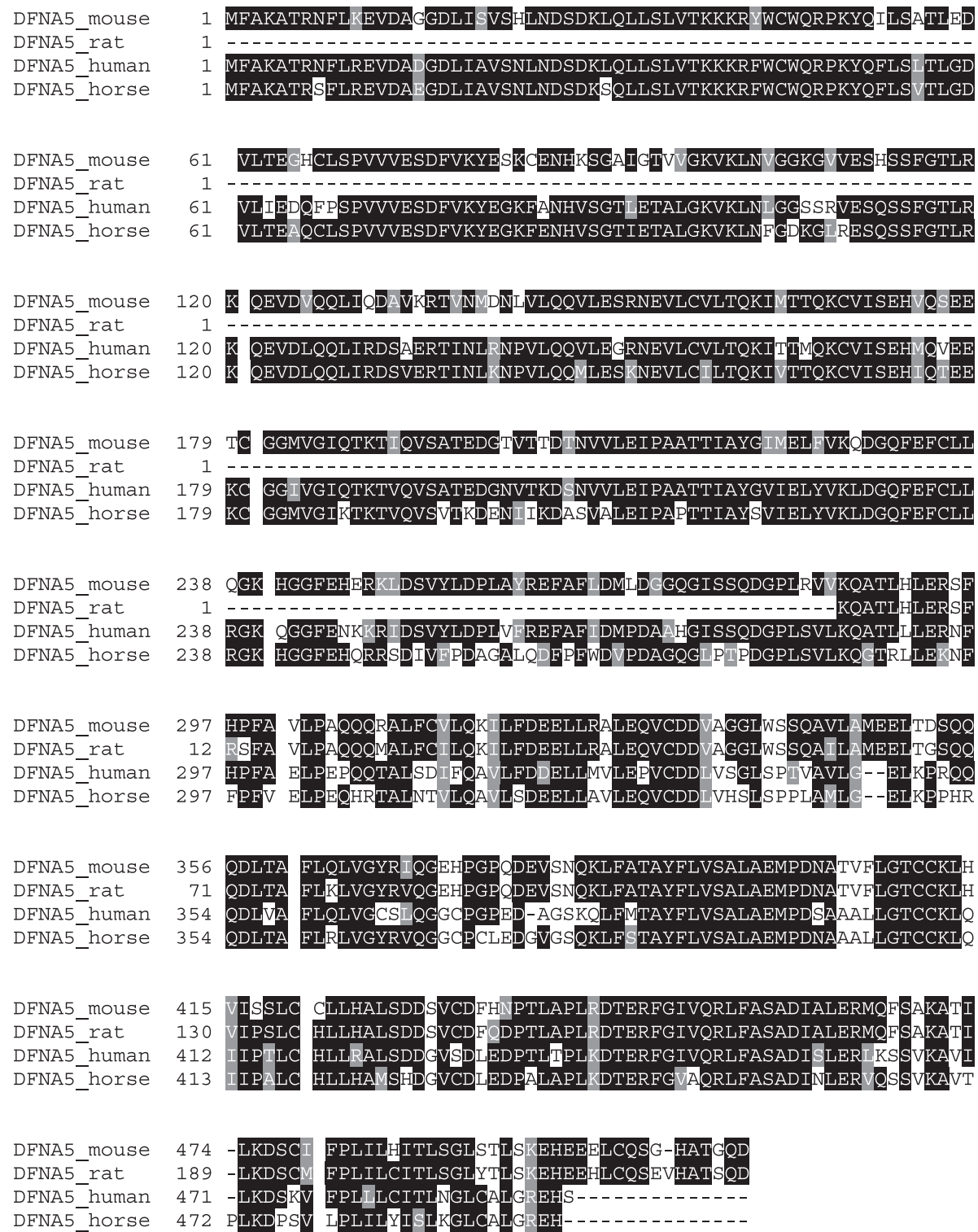

DFNA5 mouse DFNA5 rat DFNA5 human DFNA5_horse

DFNA5_mouse DFNA5 rat DFNA5 human DFNA5 horse

DFNA5 mouse DFNA5 rat DFNA5 human DFNA5_horse

DFNA5_mouse DFNA5 rat DFNA5 human DFNA5 horse

DFNA5 mouse DFNA5 rat DFNA5 DFNA5 horse 238

DFNA5_mouse DFNA5 rat DFNA5 huma DFNA5_horse

MFAKATRNFLKEVDAGGDLI SVSHLNDSDKLQLLSLVTKKKRYWCWQRPKYQILSATLED MFAKATRSFLREVDAFGDLIAVSNLNDSDKSQLLSLVTKKKRFWCWQRPKYQFLSVTLGD

QEVDVQQLIQDAVKRTVNMDNLVLQQVLESRNEVLCVLTQKIMTTQKCVISEHVQSEE QEVDLQQLIRDSAERTINLRNPVLQQVLEGRNEVLCVLTQKITTMQKCVISEHMQVEE
QEVDLQQLIRDSVERTINLKNPVLQQMLESKNEVLCILTQKIVTTQKCVISEHIQTEE

C GMVGIQTKTIQVSATEDGTVTTDTNVVLEIPAATTIAYGIMELFVKODGQFEFCLL

KC GCTVGTQTKTVQVSATEDGNVTKDSNVVT EI PAATTIAYGVIETYVKTDGQFEFCLT GGMVGIKTKTVQVSVTKDENI IKDASVALEIPAPTTIAYSVIELYVKLDGQFEFCLL

Fig. 2. Multiple alignment of DFNA5 proteins. DFNA5 homologues from human (Swiss-Prot: O60443), mouse (Swiss-Prot: Q9Z2D3), horse (GenBank: AY194290) and partial sequence of the rat (GenBank: AY194291) were aligned using CLUSTAL W [22]. Identical amino acid residues are shaded in black and similar amino acid residues are in gray shades. 
DFNA5 proteins were heterologously expressed in a wild-type $S$. pombe strain P138 (ade6-M210 leu1-32 ura4D18 $\left.h^{-}\right)$.

\subsection{Reverse transcription-polymerase chain reaction (RT- $P C R)$ assay}

RT-PCR were performed with total cellular RNA, avian myeloblastosis virus reverse transcriptase and Tfl DNA polymerase (Promega) according to the manufacturer's protocol.

Two oligonucleotide primers, DFNA5 $5^{\prime}$ (atgtttgccaaagcaaccagg) and DFNA5 $3^{\prime}$ (tcagagatcacacacttctgc), were used to amplify a 515-bp RT-PCR product corresponding to the human DFNA5 mRNA. The identity of the DFNA5 RT-PCR product was confirmed by sequencing.

\section{Results}

\subsection{Sequence analysis}

We used PROSITE [14] to search SWISS-PROT, TrEMBL and PDB databases using a consensus sequence of the $\mathrm{CCCH}$ domains of $\mathrm{Mcm} 10$ proteins as a PROSITE entry. Apart from known Mcm10 homologues, we identified the human DFNA5 protein and its mouse homologue Dfna5h (Fig. 1). Interestingly, the mutation associated with nonsyndromic hearing impairment results in the premature termination of the DFNA5 open reading frame, so that the truncated protein is lacking a region containing the $\mathrm{CCCH}$ domain [1]. Iterative PSI-BLAST searches [15] with the $\mathrm{CCCH}$ domains did not detect any significant homologies other than DFNA5, Dfna5h and members of the Mcm10 family.

To further characterize the DFNA5 proteins, we identified additional DFNA5 homologues. Screening one million plaques from a horse skin cDNA library resulted in eight positive clones. Three of these clones were chimeric, four contained partial horse DFNA5 mRNA and one contained the complete horse DFNA5 mRNA (2219 bp; GenBank accession number AY194290). The horse DFNA5 protein showed $75.0 \%$ similarity with human and $66.6 \%$ similarity with mouse DFNA5.

Only one partial cDNA clone resulted after screening $10^{6}$ clones from a rat brain cDNA library (1515 bp; GenBank accession number AY194291). The rat brain library was rescreened using a rat-specific DFNA5 probe. In addition, 5 -RACE experiments were performed on the rat brain library. However, we did not succeed in generating a fulllength rat DFNA5 clone. The partial rat DFNA5 protein showed $90.7 \%$ similarity with mouse, $64.0 \%$ with human and $60.4 \%$ with horse DFNA5 (Fig. 2). Both rat and horse DFNA5 homologues share the domain of homology with the Mcm10 family (Fig. 1).

Nuclear localization has been shown experimentally for the human, Xenopus, S. pombe and S. cerevisiae Mcm10 proteins [16]. Subcellular localization of DFNA5 proteins has not been determined yet, but both human DFNA5 and mouse Dfna5h as well as horse DFNA5 proteins share a stretch of positively charged amino acid residues (KKKR) near their $\mathrm{N}$ termini, which resemble a basic core of the monopartite nuclear localization signal $\mathrm{K}(\mathrm{K} / \mathrm{R}) \mathrm{X}(\mathrm{K} / \mathrm{R})$ [17]. Although PSORT II prediction identified a "pat4" type of nuclear localization signal in all DFNA5 homologues, it predicted the cytoplasmic localization of DFNA5 proteins as the most likely [18]. Therefore, the subcellular localization of DFNA5 proteins will need to be experimentally determined.

\subsection{Heterologous expression of DFNA5 genes in yeast}

To further characterize DFNA5, we have cloned wildtype alleles of the mouse Dfna5h and human DFNA5 as well as the mutant DFNA5 allele from DFNA5 patients and expressed them in the fission yeast $S$. pombe under a control of regulatable $n m t 1$ promoter [19]. The $n m t 1$ promoter can be repressed in the presence of thiamine in the growth medium and it can be induced by removing the thiamine. While heterologous expression of the human and mouse DFNA5 proteins did not result in any apparent phenotype, expression of the mutant DFNA5 allele caused loss of viability of the yeast cells as assessed from growth on plates lacking thiamine (Fig. 3A). We also observed a reduction in

Fig. 3. Heterologous expression of the DFNA5 proteins in fission yeast. (A) Wild-type strain (P138) transformed with either empty vector pREP3x (pREP), or construct containing mouse Dfna5h-pREP3x-mDFNA5 (DFNA5mouse), or human wild-type DFNA5 — pREP3x-hDFNA5 (DFNA5) or mutant allele of human DFNA5 - pREP3x-hDFNA5 ${ }^{\text {mut }}$ (DFNA5 ${ }^{\text {mut }}$ ) were grown on EMM plates with or without added thiamine for 3 days at $32{ }^{\circ} \mathrm{C}$. (B) Wild-type strains

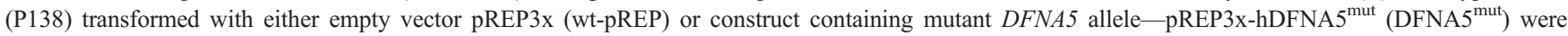
grown in EMM media without thiamine to induce expression of the mutant DFNA5 gene for the indicated time. Aliquots were taken and plated onto YE3S plates to determine viability. Percentage of relative viability was calculated as (number of colonies at time $=t$ since thiamine withdrawal) $100 /($ number of colonies at $t=18 \mathrm{~h}$ since thiamine withdrawal). Values represent averages of two experiments. (C) Flow cytometry analysis of the strains described for panel A. Aliquots for flow cytometry were taken at indicated time since thiamine withdrawal. (D) Light microscopy images of ethanol-fixed cells as described for panel A taken at indicated time since thiamine withdrawal. Left-hand panels show phase images and right-hand panels show DAPI staining of DNA. (E) RT-PCR assays detecting expression of wild-type and mutant alleles of the human DFNA5 heterologously expressed in the yeast cells. RT-PCR assays were performed with total RNA isolated from the wild-type yeast strain P138 (wt) (lanes 1-2) or the P138 strain transformed with either empty vector pREP3x (pREP) (lanes 3-4) or pREP3x-hDFNA5 construct (DFNA5) (lanes 5-10) or pREP3x-DFNA5 ${ }^{\text {mut }}$ construct (DFNA5 ${ }^{\text {mut }}$ ) (lanes $11-16$ ). Strains were grown in EMM medium with or without thiamine for $20 \mathrm{~h}$. RT-PCR products obtained after 25 or 28 cycles of PCR amplification are shown. RT-PCR products are also seen under + thiamine conditions (lanes 5,6,11,22) as the $n m t 1$ promoter is known to be leaky when repressed with thiamine. Control reactions after 28 cycles of PCR amplification where no reverse transcriptase was added ( - RT) to exclude DNA contamination in the samples are shown. DNA size marker (1-kb DNA ladder, Invitrogen) is given in lane $17(\mathrm{M})$. 
A

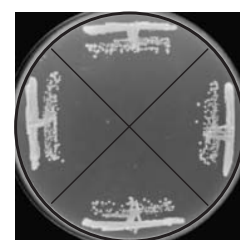

+thiamine

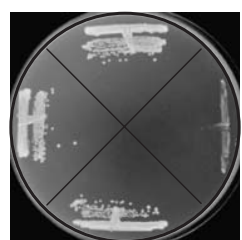

-thiamine

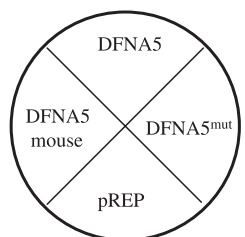

B

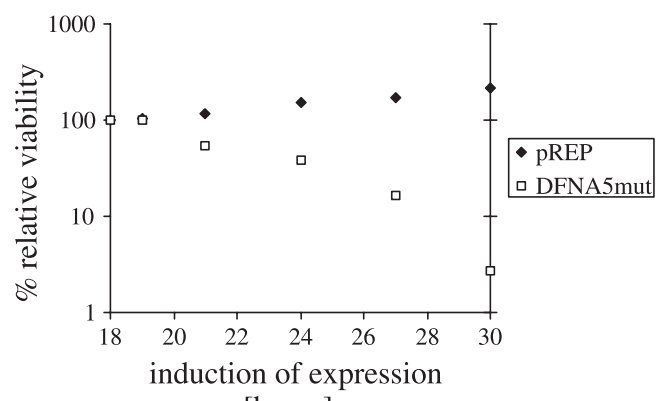
[hours]

C

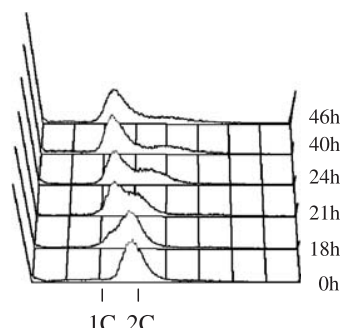
DFNA5 ${ }^{\text {mut }}$ - thiamine

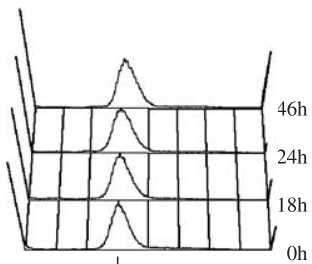

$2 \mathrm{C}$

DFNA5 - thiamine

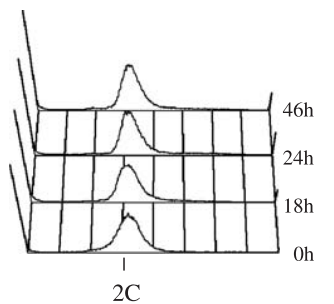

DFNA5 $5^{\text {mut }}+$ thiamine

$\mathrm{D}$

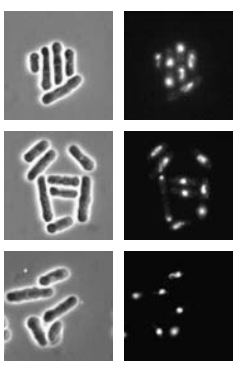

DFNA5 $5^{\text {mut }}$

(24h-thiamine)

DFNA5 ${ }^{\text {mut }}$

(30h-thiamine)

pREP

(30h-thiamine)

$\mathrm{E}$

$\begin{array}{lllllllllllllllll}1 & 2 & 3 & 4 & 5 & 6 & 7 & 8 & 9 & 10 & 11 & 12 & 13 & 14 & 15 & 16 & \mathrm{M}\end{array}$

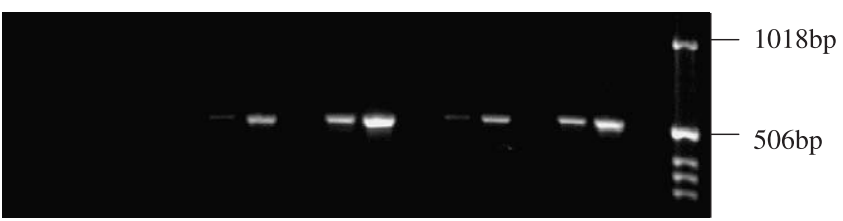

$\begin{array}{lllllllllllllllllll}28 & 28 & 28 & 28 & 25 & 28 & \text {-RT } & 25 & 28 & \text {-RT } & 25 & 28 & \text {-RT } & 25 & 28 & \text {-RT }\end{array}$

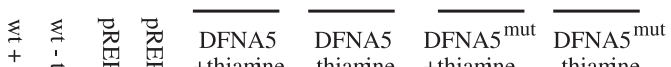

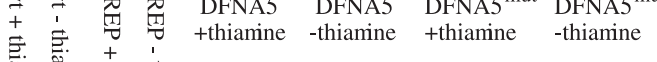

突 
viability in exponentially growing cells after transfer to medium lacking thiamine (Fig. 3B). Flow cytometry of exponentially growing wild-type haploid fission yeast cells shows only peak corresponding to $2 \mathrm{C}$ DNA content. This is because cells enter $\mathrm{S}$ phase before cytokinesis is completed. Flow cytometry analysis showed that yeast cells expressing
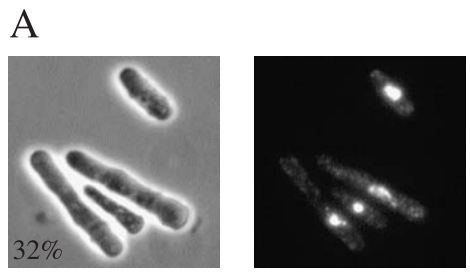

Cdc23-M36-td-DFNA5 ${ }^{\text {mut }}$ -thiamine
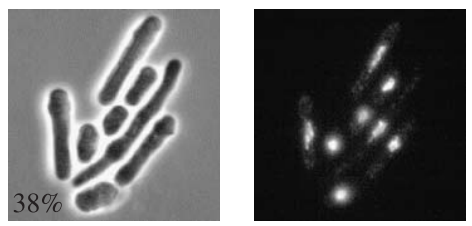

Cdc23-IE2-td-DFNA5 ${ }^{\text {mut }}$ -thiamine
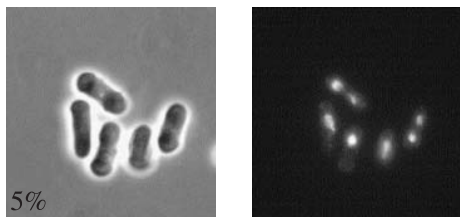

Cdc23-M36-td

-thiamine
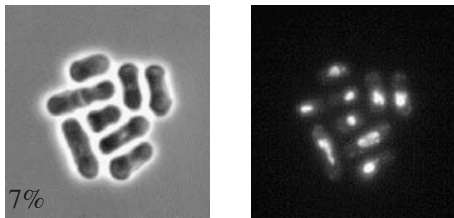

Cdc23-IE2-td

-thiamine
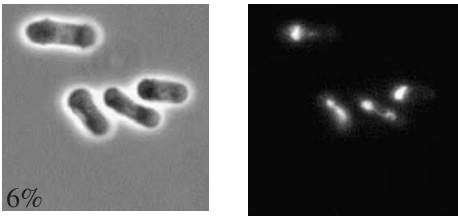

Cdc23ts-M36-td-DFNA5 ${ }^{\text {mut }}$ + thiamine
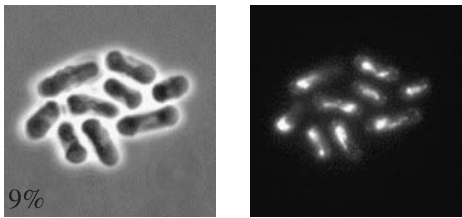

Cdc23-IE2-td-DFNA5 ${ }^{\text {mut }}$ + thiamine

B

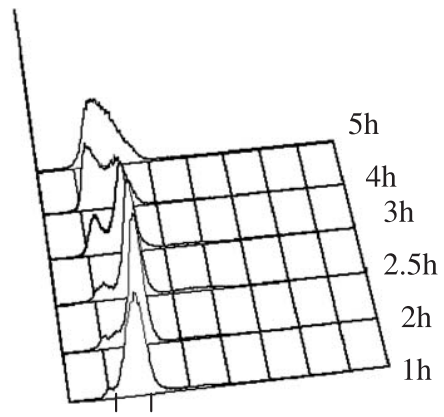

$1 \mathrm{C} 2 \mathrm{C}$

cdc23-M36-td - $37^{\circ} \mathrm{C}$

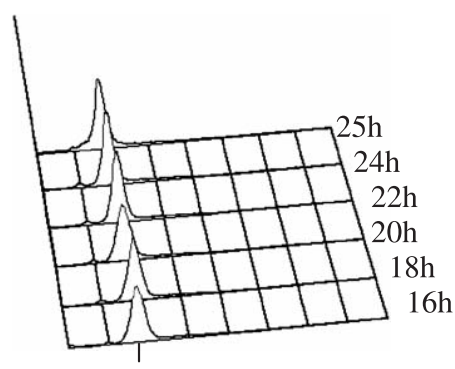

$2 \mathrm{C}$

$c d c 23-M 36-t d-25^{\circ} \mathrm{C}$

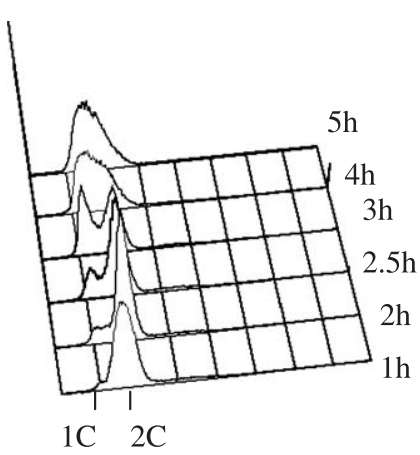

$c d c 23-M 36-t d$-DFNA5 ${ }^{\text {mut }}-37^{\circ} \mathrm{C}$

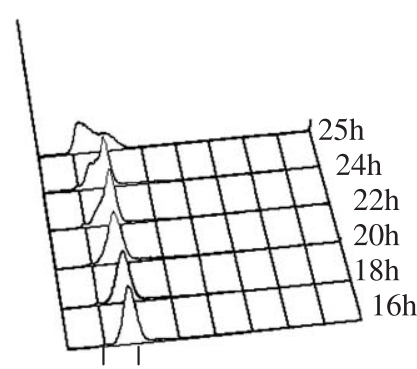

$1 \mathrm{C} \quad 2 \mathrm{C}$

$c d c 23-M 36-t d$-DFNA $5^{\text {mut }}-25^{\circ} \mathrm{C}$

Fig. 4. Mutant DFNA5 allele heterologously expressed in fission yeast has synthetic phenotype with $c d c 23$ mutant. (A) $c d c 23-M 36-t d$ and $c d c 23-I E 2-t d$ strains containing temperature-sensitive alleles of $c d c 23$ and transformed with either empty vector pREP3x ( $c d c 23-M 36-t d$, $c d c 23-I E 2-t d$ ) or construct containing mutant DFNA5 allele-pREP3x-hDFNA5 ${ }^{\text {mut }}$ (cdc23tstd-DFNA5 ${ }^{\text {mut }}, c d c 23-I E 2-t d$-DFNA5 ${ }^{\text {mut }}$ ) were grown on EMM with or without thiamine at permissive temperature, $25{ }^{\circ} \mathrm{C}$, for $24 \mathrm{~h}$ and fixed with ethanol. Left-hand panels show phase images and right-hand panels show DAPI staining of DNA. Percentage of elongated cells (longer than $15 \mu \mathrm{M}$ ) is shown. (B) Flow cytometry analysis of the $c d c 23-M 36-t d$ strain transformed with either empty vector pREP3x ( $c d c 23-$ M36- $t d$ ) or construct containing mutant DFNA5 allele-pREP3x-hDFNA5 ${ }^{\text {mut }}\left(c d c 23 t s t d\right.$-DFNA5 $\left.{ }^{\text {mut }}\right)$. Strains were grown in EMM media without thiamine at permissive temperature $\left(25^{\circ} \mathrm{C}\right)$ for $16 \mathrm{~h}$ to induce expression of the mutant $D F N A 5$. The cultures were then split and one half was shifted to nonpermissive temperature $\left(37^{\circ} \mathrm{C}\right)$ to inactivate the $\mathrm{Cdc} 23$ protein. Aliquots for flow cytometry were taken at indicated time. 
the mutant DFNA5 arrested in cell cycle with approximately 1C DNA content, suggesting that the cells were blocked in G1 or early $\mathrm{S}$ phase (Fig. 3C). Interestingly, this is the phenotype associated with $m \mathrm{~cm} 10$ mutants $[5,8]$. However, unlike many fission yeast mutants blocked in DNA replication, cells expressing mutant DFNA5 did not elongate significantly (less than $5 \%$ of cells were elongated) and arrested cells did not show a "cut" phenotype where septation and/or cytokinesis occur in the absence of normal sister chromatid separation (Fig. 3D). We used an RT-PCR assay to confirm that both the wild-type and the mutant alleles of human DFNA5 are expressed in the yeast cells upon induction (Fig. 3E).

\subsection{Genetic interactions between DFNA5 and mcm 10}

Following a possible link between DFNA5 and Mcm10 proteins, we tested the effect of DFNA5 expression in a fission yeast $m c m 10$ homologue, the $c d c 23$ mutant. Neither human nor mouse DFNA5 proteins were able to complement a fission yeast $c d c 23$ mutant (not shown), which is not surprising since the proteins do not share significant similarity outside of the CCCH domain. When mutant DFNA5 was expressed in a $c d c 23$ temperature-sensitive degron mutant strains ( $c d c 23-M 36-t d, c d c 23-I E 2-t d$ ), even at the permissive temperature there was a significantly higher number of elongated cells compared to strains not expressing the mutant DFNA5 (Fig. 4A). Elongated cells indicate a cell cycle block, which uncouples growth from cell cycle events (e.g. block in chromosomal DNA replication). Moreover, when mutant DFNA5 expression was induced in a $c d c 23-M 36-t d$ mutant and subsequently shifted to nonpermissive temperature, cells arrested with unreplicated DNA earlier than strains not expressing mutant DFNA5 allele (Fig. 4B). Therefore, in fission yeast, heterologous expression of the mutant DFNA5 allele has a synthetic phenotype with the $c d c 23$.

\section{Discussion}

Here we describe the first model system for studying molecular effects caused by the mutation in the DFNA5 gene, which was found to be responsible for nonsyndromic hearing impairment. Comparison of the wild-type and the mutant DFNA5 alleles heterologously expressed in yeast allowed us to examine a phenotype associated with a dominant negative or gain of function effect of the DFNA5 mutant. We provide several lines of evidence suggesting a possible link between the DFNA5 and Mcm10 proteins. Firstly, we identified a domain of homology shared between the Mcm10 family of replication proteins and members of the DFNA5 family, including the newly described horse and rat DFNA5 homologues. The function of the $\mathrm{CCCH}$ domain remains obscure, but its importance is underlined by the fact that it is essential for the function of Mcm10 proteins. In addition, this domain is absent in the dominant DFNA5 mutant resulting in hearing impairment. Secondly, we found that heterologous expression of the mutant DFNA5 in fission yeast leads to a block in G1 or early S phase of the cell cycle with approximately 1C DNA content. Furthermore, expression of the mutant DFNA5 in the yeast $c d c 23$ mutant has a synthetic growth defect and the arrest caused by $c d c 23$ inactivation is faster when the mutant DFNA5 is induced. The observed effect could be either direct or indirect and it is possible that there are other currently unidentified cellular processes affected by the mutant DFNA5 allele.

Could DFNA5 have a function related to the DNA replication function of $\mathrm{Mcm} 10$ ? This cannot be strongly predicted since the proteins do not share significant similarity outside of the $\mathrm{CCCH}$ domain and it is unlikely that DFNA5 is a distant homologue of Mcm10 proteins. However, it is of interest that the reduction in DFNA5 expression can contribute to the etoposide resistance in human melanoma cells. The etoposide-resistant phenotype may be due to an increased cellular susceptibility to drug-induced apoptosis [3]. Etoposide is used as an antineoplastic agent and causes DNA strand breakage via inhibition of DNA topoisomerase II. This could implicate DFNA5 in some aspect of the response to DNA damage. Indeed, alterations in DNA repair proteins have been observed in drug-resistant melanoma lines [20,21]. Alternatively, DFNA5 and Mcm10 proteins may have unrelated functions, but share the $\mathrm{CCCH}$ domain to perform an activity unrelated to DNA replication. Further experiments are required to clarify the function of DFNA5. Our fission yeast model now provides a tool to examine the molecular basis underlying the DFNA5 hearing impairment in human.

\section{Acknowledgements}

This work was supported by a grant from the Cancer Research UK.

LVL holds a research position with the Flemish FWO (Fonds voor Wetenschappelijk Onderzoek). This research was supported by a grant of the GSKE (Geneeskundige Stichting Koningin Elisabeth) to GVC and LVL, and by a grant of the University of Antwerp to LVL.

The construction of the horse cDNA library was funded in part by The United Professional Horsemen's Association, The American Saddlebred Horse Association and The Belgian Draft Horse Corporation.

We thank Lynne Larkman for technical assistance.

\section{References}

[1] L. Van Laer, E.H. Huizing, M. Verstreken, D. van Zuijlen, J.G. Wauters, P.J. Bossuyt, P. Van de Heyning, W.T. McGuirt, R.J. Smith, P.J. Willems, P.K. Legan, G.P. Richardson, G. Van Camp, Nonsyndromic hearing impairment is associated with a mutation in DFNA5, Nat. Genet. 20 (1998) 194-197. 
[2] D.A. Thompson, R.J. Weigel, Characterization of a gene that is inversely correlated with estrogen receptor expression (ICERE-1) in breast carcinomas, Eur. J. Biochem. 252 (1998) 169-177.

[3] H. Lage, H. Helmbach, C. Grottke, M. Dietel, D. Schadendorf, DFNA5 (ICERE-1) contributes to acquired etoposide resistance in melanoma cells, FEBS Lett. 494 (2001) 54-59.

[4] N.A. Solomon, M.B. Wright, S. Chang, A.M. Buckley, L.B. Dumas, R.F. Gaber, Genetic and molecular analysis of DNA43 and DNA52: two new cell-cycle genes in Saccharomyces cerevisiae, Yeast 8 (1992) $273-289$.

[5] A.M. Merchant, Y. Kawasaki, Y. Chen, M. Lei, B.K. Tye, A lesion in the DNA replication initiation factor $\mathrm{Mcm} 10$ induces pausing of elongation forks through chromosomal replication origins in Saccharomyces cerevisiae, Mol. Cell. Biol. 17 (1997) 3261-3271.

[6] Y. Kawasaki, S. Hiraga, A. Sugino, Interactions between Mcm10p and other replication factors are required for proper initiation and elongation of chromosomal DNA replication in Saccharomyces cerevisiae, Genes Cells 5 (2000) 975-989.

[7] J.A. Wohlschlegel, S.K. Dhar, T.A. Prokhorova, A. Dutta, J.C. Walter, Xenopus Mcm10 binds to origins of DNA replication after $\mathrm{Mcm} 2-7$ and stimulates origin binding of Cdc45, Mol. Cell. 9 (2002) 233-240.

[8] S.J. Aves, N. Tongue, A.J. Foster, E.A. Hart, The essential Schizosaccharomyces pombe $c d c 23$ DNA replication gene shares structural and functional homology with the Saccharomyces cerevisiae DNA43 (MCM10) gene, Curr. Genet. 34 (1998) 164-171.

[9] M. Lei, Characterization of a new DNA replication initiation factor, Mcm10, Yeast 18 (2001) S46.

[10] L. Homesley, M. Lei, Y. Kawasaki, S. Sawyer, T. Christensen, B.K. Tye, Mcm10 and the MCM2-7 complex interact to initiate DNA synthesis and to release replication factors from origins, Genes Dev. 14 (2000) 913-926.

[11] S. Moreno, A. Klar, P. Nurse, Molecular genetic analysis of fission yeast Schizosaccharomyces pombe, Methods Enzymol. 194 (1991) $795-823$.
[12] K. Lindner, J. Gregan, S. Montgomery, S.E. Kearsey, Essential role of MCM proteins in premeiotic DNA replication, Mol. Biol. Cell 13 (2002) 435-444.

[13] L.D. Lieto, E.G. Cothran, Characterization of expressed sequence tags generated from skin cDNA clones of Equus caballus by single pass sequencing, Anim. Biotechnol. 12 (2001) 87-97.

[14] K. Hofmann, P. Bucher, L. Falquet, A. Bairoch, The PROSITE database, its status in 1999, Nucleic Acids Res. 27 (1999) 215-219.

[15] S.F. Altschul, T.L. Madden, A.A. Schaffer, J. Zhang, Z. Zhang, W. Miller, D.J. Lipman, Gapped BLAST and PSI-BLAST: a new generation of protein database search programs, Nucleic Acids Res. 25 (1997) 3389-3402.

[16] M. Lei, B.K. Tye, Initiating DNA synthesis: from recruiting to activating the MCM complex, J. Cell. Sci. 114 (2001) 1447-1454.

[17] M.R. Hodel, A.H. Corbett, A.E. Hodel, Dissection of a nuclear localization signal, J. Biol. Chem. 276 (2001) 1317-1325.

[18] K. Nakai, P. Horton, PSORT: a program for detecting sorting signals in proteins and predicting their subcellular localization, Trends Biochem. Sci. 24 (1999) 34-36.

[19] K. Maundrell, Thiamine-repressible expression vectors pREP and pRIP for fission yeast, Gene 123 (1993) 127-130.

[20] H. Lage, M. Christmann, M.A. Kern, M. Dietel, M. Pick, B. Kaina, D. Schadendorf, Expression of DNA repair proteins hMSH2, hMSH6, hMLH1, O6-methylguanine-DNA methyltransferase and $N$-methylpurine-DNA glycosylase in melanoma cells with acquired drug resistance, Int. J. Cancer 80 (1999) 744-750.

[21] H. Lage, H. Helmbach, M. Dietel, D. Schadendorf, Modulation of DNA topoisomerase II activity and expression in melanoma cells with acquired drug resistance, Br. J. Cancer 82 (2000) 488-491.

[22] J.D. Thompson, D.G. Higgins, T.J. Gibson, CLUSTAL W: improving the sensitivity of progressive multiple sequence alignment through sequence weighting, position-specific gap penalties and weight matrix choice, Nucleic Acids Res. 22 (1994) 4673-4680. 